\title{
Intravascular Ultrasound (IVUS): A Potential Arthroscopic Tool for Quantitative Assessment of Articular Cartilage
}

\author{
Yan-Ping Huang ${ }^{1}$ and Yong-Ping Zheng ${ }^{* 1,2}$ \\ ${ }^{1}$ Department of Health Technology and Informatics, The Hong Kong Polytechnic University, Hong Kong, China \\ ${ }^{2}$ Research Institute of Innovative Products and Technologies, The Hong Kong Polytechnic University, Hong Kong, \\ China
}

\begin{abstract}
Conventional ultrasound examination of the articular cartilage performed externally on the body surface around the joint has limited accuracy due to the inadequacy in frequency used. In contrast to this, minimally invasive arthroscopy-based ultrasound with adequately high frequency may be a better alternative to assess the cartilage. Up to date, no special ultrasound transducer for imaging the cartilage in arthroscopic use has been designed. In this study, we introduced the intravascular ultrasound (IVUS) for this purpose. An IVUS system with a catheter-based probe $(\phi \approx 1 \mathrm{~mm})$ was used to measure the thickness and surface acoustical reflection of the bovine patellar articular cartilage in vitro before and after degeneration induced by enzyme treatments. Similar measurement was performed using another high frequency ultrasound system (Vevo) with a probe of much larger size and the results were compared between the two systems. The thickness measured using IVUS was highly correlated $(r=0.985, p<0.001)$ with that obtained by Vevo. Thickness and surface reflection amplitude measured using IVUS on the enzymatically digested articular cartilage showed changes similar to those obtained by Vevo, which were expectedly consistent with previous investigations. IVUS can be potentially used for the quantitative assessment of articular cartilage, with its ready-to-use arthroscopic feature.
\end{abstract}

Keywords: Ultrasound, high frequency, intravascular ultrasound (IVUS), articular cartilage, thickness, acoustical properties, degeneration, osteoarthritis, enzyme digestion.

\section{INTRODUCTION}

Ultrasound has been widely adopted to assess articular cartilage, due to its non-invasiveness and easy access with acceptably low cost [1-4]. For example, loss of the sharpness of the cartilage surface and the clarity of the cartilage layer, narrowing of the cartilage thickness were typical observations from the osteoarthritic cartilages $[1,3]$. However, as articular cartilage is a thin tissue (typical of $1 \sim 3 \mathrm{~mm}$ in thickness in the human knee joints [5]), it brings an inherent constraint between the imaging spatial resolution and the penetration depth to the conventional ultrasound assessment performed externally from the joint surface. Due to the existence of superficial layers (skin, muscle or synovial fluid etc.) that the ultrasound has to penetrate prior to the cartilage, too high frequency cannot be chosen, thus limiting the accuracy of measurement. Difficulties in performing external ultrasound examination also include the restrictions in testing postures, probe positions and orientations [1, 4]. For example, a full flexion of the knee for

*Address correspondence to this author at the Department of Health Technology and Informatics, The Hong Kong Polytechnic University, Hong Kong, China; Tel: 852 27667664; Fax: 852 23624365;

E-mail: ypzheng@ieee.org

Yan-Ping Huang is currently working as a $\mathrm{PhD}$ candidate in the Department of Health Technology and Informatics, The Hong Kong Polytechnic University, Hong Kong, China.

Yong-Ping Zheng is currently Professor of the Department of Health Technology and Informatics and Associate Director of the Research Institute of Innovative Products and Technologies, The Hong Kong Polytechnic University, Hong Kong, China. better ultrasound imaging will not be possible for some osteoarthritic patients with knee pain. Therefore, more and more researchers have become interested in a minimally invasive but more direct measurement of the articular cartilage using arthroscopy-based techniques [6, 7]. According to statistics, arthroscopy of the knee was the seventh most frequent operation in 1994 in USA [8] and arthroscopy had been also a common outpatient operation performed by rheumatologists under local or regional anaesthesia [9]. Ultrasound transducers fixed with the arthroscopic device, possibly with the combination of other techniques, can be used to measure a variety of articular cartilage characteristics such as the thickness, acoustical and mechanical properties in vivo. Recently, extensive investigations in basic research on osteoarthritis using high frequency ultrasound have been performed [6, 10-13] and it was found that osteoarthritic cartilages did show changes in these properties such as the acoustical surface reflection and the stiffness.

Researchers have previously reported the adoption of single element ultrasound transducers with a diameter of $3 \sim 4$ $\mathrm{mm}$ for the arthroscopic measurement [6,7], but arthroscopic imaging has not been reported in vivo. The systems used in pilot studies on imaging the articular cartilage [14, 15] were not proper for arthroscopic applications due to a limited intra-articular space. Therefore, special design is necessary to apply the ultrasound transducer for the intraarticular imaging and evaluation of articular cartilage. High frequency $(>20 \mathrm{MHz})$ intravascular ultrasound (IVUS), which is a mature device originally designed for measuring the morphology of vasculature in cardiology $[16,17]$, has a 
small profile with respect to the catheter probe. The catheter probe is capable of imaging a cross-sectional part of the blood vessels even under the very limited space with intravascular operation, so we think it may be potentially applicable (or at least at the current stage) for arthroscopic assessment and monitoring of articular cartilage with improved accuracy compared to the low frequency $(<10 \mathrm{MHz})$ single element ultrasound transducers. IVUS could be potentially incorporated in a separate channel with the assist from the arthroscopic view of the operation. Therefore, we initiated in this study using the IVUS as a new tool to measure the articular cartilage properties along with 2-D ultrasound imaging. As a first step towards this direction, we aimed in this paper to demonstrate the capability of this method in measuring the thickness of the cartilage and in quantifying the change of surface acoustical reflection induced by enzyme digestions in vitro.

\section{MATERIALS AND METHODOLOGY}

\subsection{Measurement Systems}

An IVUS real-time imaging system (In-Vision Gold, Volcano, San Diego, CA, USA) with a catheter of 3.5F ( 1.17 mm, Fig. 1a) (REF 85900, Eagle Eye Gold, Volcano) was used in the current study. A multi-element solid-state cylindrical array transducer was installed at the tip of the catheter providing a $360^{\circ}$ view of the surrounding tissue (Fig. 2a). The central frequency of the ultrasound transducer was $20 \mathrm{MHz}$ with a spatial peak temporal average (SPTA) derated intensity of $0.354 \mathrm{~mW} / \mathrm{cm}^{2}$. Usable length of the catheter was $150 \mathrm{~cm}$. A maximum area of $16 \times 16 \mathrm{~mm}^{2}$ could be acquired from around the transducer and a setting of $10 \times 10 \mathrm{~mm}^{2}(\Phi=5 \mathrm{~mm})$ was used in the current study because it optimized the spatial resolution in the B-mode image while capable of imaging a whole thickness of the cartilage (Fig. 2a). Cross-sectional images with 8-bit precision were recorded for off-line analysis.

Images were also collected by another high frequency ultrasound system-Vevo770 (VisualSonics, Toronto, Ontario, Canada) using a scanhead with a central frequency of $55 \mathrm{MHz}$ (RMV708, VisualSonics, Fig. 1b), in order to compare with the measurement results from IVUS. The diameter of the probe was about $45 \mathrm{~mm}$ with a cover at the tip where a single element ultrasound transducer was installed (Fig. 1b). Ultrasound images were acquired by fast lateral mechanical scanning of the ultrasound transducer within the cover. The cover was firmly attached to the probe with a thin film just installed in front of the ultrasound transducer, serving as a window for passing through the ultrasound beam. The cover was injected with deionized water as a coupling media for the ultrasound. The axial and lateral resolutions of the transducer were about 30 and 70 $\mu \mathrm{m}$, respectively. The focus of the probe was $4.5 \mathrm{~mm}$ in front of the transducer. A digital radio-frequency (RF) mode with 12-bit data precision was used in the Vevo system for collecting the original $\mathrm{RF}$ data in order to increase the measurement sensitivity. A rectangular window could be selected in the B-mode ultrasound image for collecting the RF data. For this window, the location, size and the number of A-lines could be set before data collection. For the current RF setting, a lateral interval of $110 \mu \mathrm{m}$ was used for each Aline collection and would also be used for subsequent spatial averaging to measure the thickness and acoustical parameters. The location of the window was selected to include both the reflections from the surface and the bonecartilage interface.

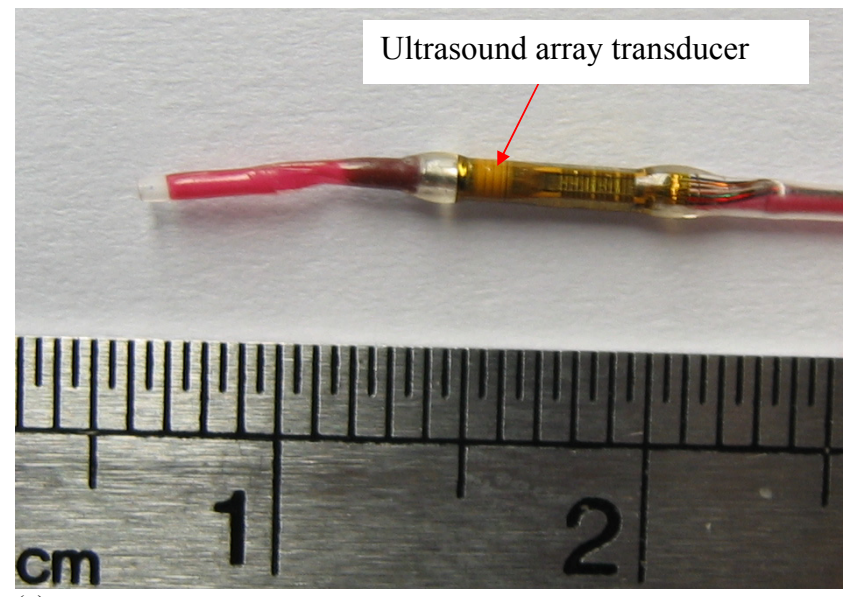

(a)

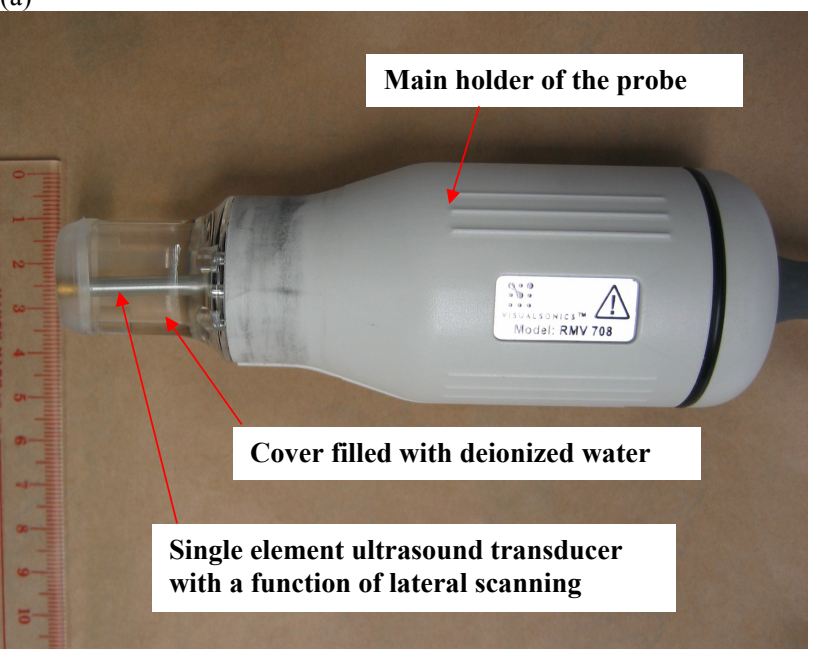

(b)

Fig. (1). Photographs of (a) the catheter tip including the ultrasound array transducer in the IVUS system and (b) the RMV708 probe in Vevo770. All the numbers shown in the scales are with a unit of centimeter.

\subsection{Experimental Tests}

Experiments were conducted on two groups of samples (Group 1 and Group 2), each group containing 8 cartilage specimens. Each specimen was a disk of cylindrical articular cartilage $(\Phi=6.35 \mathrm{~mm})$ attached to its subchondral bone excised from various quadrants of 5 bovine patellae [18]. The patellae were collected from a local market within 6 hours of sacrifice and those without obvious visual lesions were selected for preparing the disk samples. For the preparation process of the cartilage disks, please refer to our previous publication [18]. After preparation, the specimens were wrapped in gauze soaked with physiological saline solution $\left(0.15 \mathrm{M}\right.$ in concentration) and stored at $-20^{\circ} \mathrm{C}$ before testing. At the day of testing, the specimens were first thawed in physiological saline solution for $1.5 \mathrm{~h}$ before ready for the ultrasound experiments. Each specimen was fixed 
using plasticine in a container to ensure a horizontal surface for performing the scanning. During the scanning process, the sample was also immersed in physiological saline solution to minimize the effect of shrinking or swelling of the cartilages and the IVUS catheter tip was placed manually over the center of the sample for the imaging process. For each sample, three repeated measurements were conducted and the averaged value was used to represent the tissue properties of the tested sample. For the measurement using the Vevo system, similar procedures were performed except that the probe was fixed vertically by a clamp which could be adjusted in the vertical and lateral directions for the ease of the scanning operation. Special attention had been paid to get the maximum signal reflected from the cartilage surface in all the scans as this would affect the amplitude of the reflection measurement. After the scanning using IVUS and Vevo, samples in Group 1 were digested with $0.25 \%$ trypsinEDTA solution (GIBCO, Invitrogen, Carlsbad, CA, USA) to mainly deplete the proteoglycan (PG) [19] for $5 \mathrm{~h}$ at room temperature $\left(25 \pm 1^{\circ} \mathrm{C}\right)$, while those in Group 2 were treated with $30 \mathrm{U} / \mathrm{ml}$ collagenase solution (GIBCO, Invitrogen, Carlsbad, CA, USA) at $37{ }^{\circ} \mathrm{C}$ to mainly digest the collagen network [20] for the same period of $5 \mathrm{~h}$. After the enzyme digestions, the samples were then immersed in physiological solution for $1 \mathrm{~h}$ in order to allow the digestion process to be stabilized. Then the scanning process was repeated again using IVUS and Vevo. For each scan, the distance between the ultrasound probes and the cartilage surface was kept roughly the same by observing the location of the cartilage surface from B-mode images and all other configurations such as gain and display settings of the device were set to be all the same to facilitate the inter-specimen comparisons.

\subsection{Data Processing Method and Statistics}

A program with graphic user interface (GUI) written in Matlab (2007a, MathWorks, Natick, MA, USA) was used to extract the two parameters: the thickness of cartilage and surface reflection amplitude of the ultrasound signals. The locations of the cartilage surface and cartilage-bone interface could be selected from the GUI by adding two windows to include the two interface reflections. The surface reflection was calculated as the maximum amplitude of the echo from the cartilage-saline interface after averaging in the horizontal direction. The thickness was calculated based on the interval between the two interface reflections after spatial averaging and the speed of sound. A region with a width of approximately $1 \mathrm{~mm}$ was used for spatial averaging to get the averaged amplitude of the interface for calculating the two parameters (Fig. 2b). In this study, the speed of sound was assumed to be $1610 \mathrm{~m} / \mathrm{s}$ in normal articular cartilage and be 0.988 and 0.984 of this value after trypsin and collagenase treatments, respectively [6]. For the IVUS measurement, a total of 384 pixels equalized to a width and length of $10 \mathrm{~mm}$ for the image and therefore each pixel would represent a length of approximately $0.027 \mathrm{~mm}$ in the depth and lateral direction in articular cartilage, which determined the resolution of the measurement using IVUS images. A-mode lines were extracted from each row data of the B-mode images. During the experiment, the cartilage surface was also deliberately aligned to be parallel with the vertical direction of the image so a row signal of the image would represent an A-line signal of ultrasound waves penetrating in the depth direction of the cartilage.

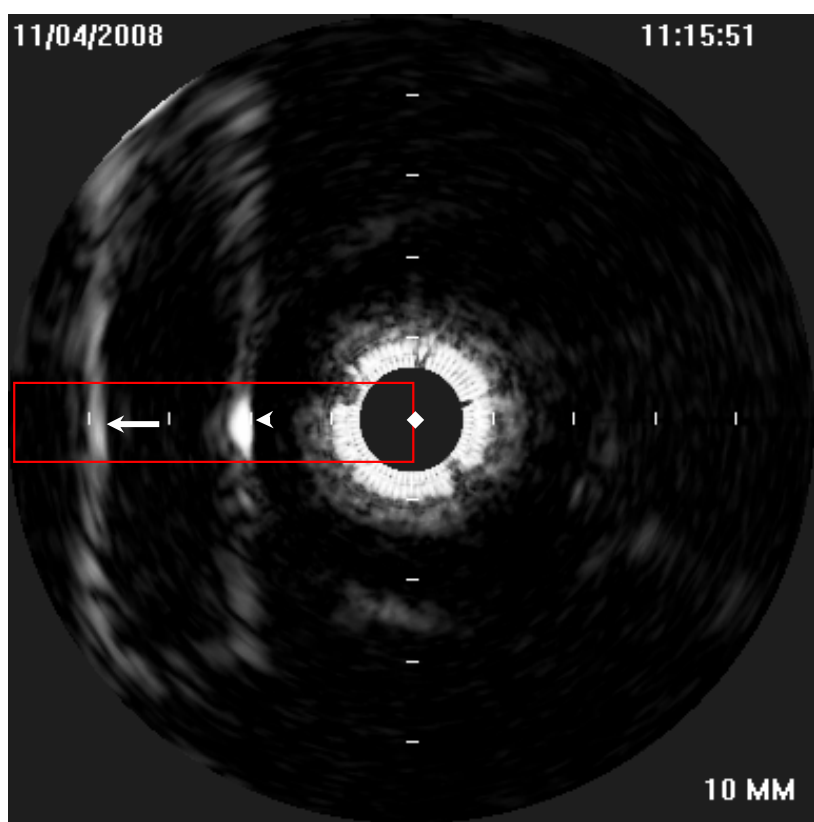

(a)

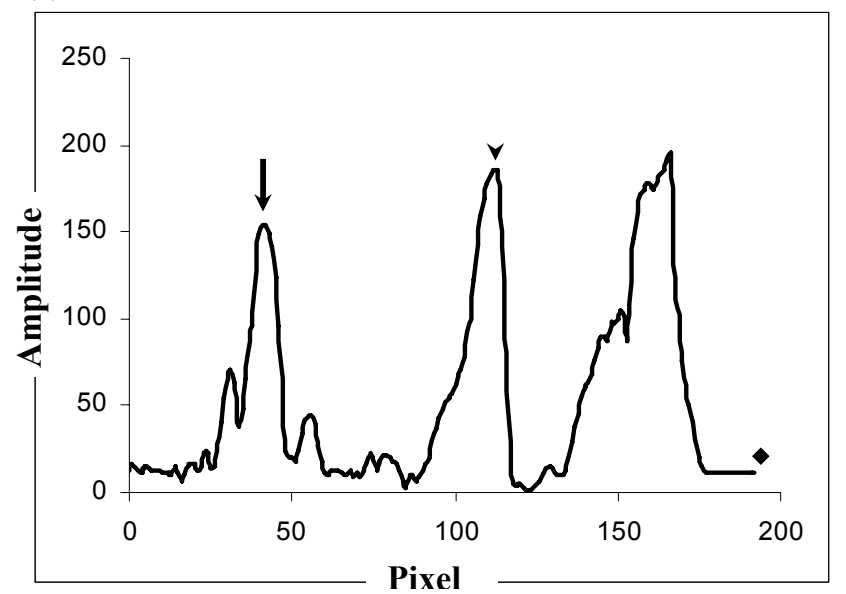

(b)

Fig. (2). (a) A typical IVUS B-mode image of articular cartilage. The two interfaces of the cartilage are shown. Arrowhead: the cartilage-saline solution interface; arrow: the cartilage-subchondral bone interface. The rectangular window shows where the average was taken for obtaining the parameters of the articular cartilage; (b) Typical interface echoes after spatial averaging in the selected region. Arrowhead: the cartilage-saline solution interface; arrow: the cartilage-subchondral bone interface; diamond: the center of the image. Unit of the axis: no.

For the measurement using Vevo, similar procedures were applied to calculate the surface reflection coefficient and thickness with caution that the ultrasonic signals were not saturated (Fig. 3). Using a sampling frequency of 420 $\mathrm{MHz}$ and a constant speed of sound of $1610 \mathrm{~m} / \mathrm{s}$, each point in the RF digital data represented a length of approximately $0.0019 \mathrm{~mm}$, which was much smaller than that of IVUS. A Hilbert transform was performed to obtain the amplitude signal for each A-line before averaging for the selected region. In order to compensate for the effect of focusing of 
the focused transducer used in the Vevo system, the surface reflection amplitude was corrected using a normalized factor obtained by a perfect reflector at the same distance [6]. For this correction method, the normalized factor at the focus was one and at other distance, it was the proportion of the amplitude compared to that at the focus.

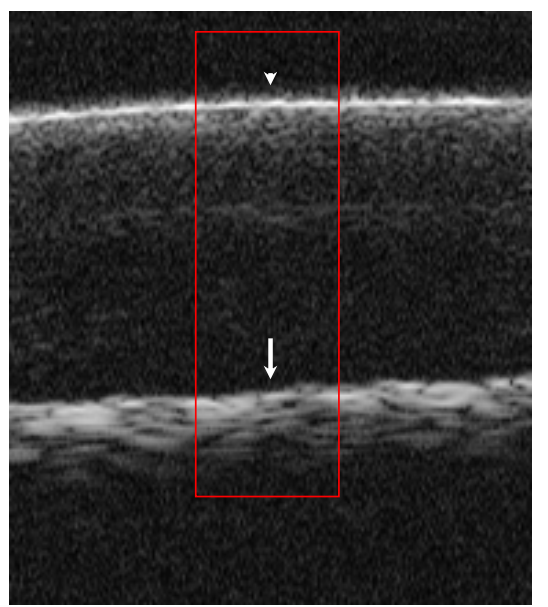

(a)

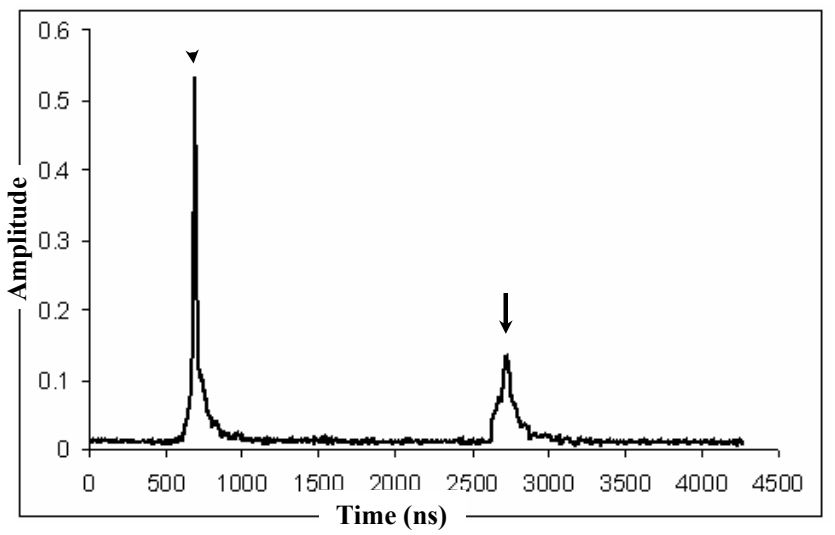

(b)

Fig. (3). (a) A typical B-mode image of articular cartilage in Vevo ultrasound imaging system. The two interfaces of the cartilage are shown. Arrowhead: the cartilage-saline solution interface; arrow: the cartilage-subchondral bone interface. The rectangular window shows where the average was taken for obtaining the parameters of the articular cartilage; (b) Typical interface echoes after spatial averaging in the selected region. Arrowhead: the cartilage-saline solution interface; arrow: the cartilage-subchondral bone interface. Unit of the y axis: arbitary.

A coefficient of variance (CV) was used to represent the repeatability of measurement, if necessary. For the thickness measurement, the values of the 16 specimens (Group $1+$ Group 2) before enzyme treatments measured from IVUS were correlated to those measured from Vevo using the Pearson correlation coefficient. As the thickness from the two measurements was both calculated from the principle of a time-of-flight technique, a significant correlation would show that the two methods were comparable except a constant difference which might come from the discrepancy in measuring the propagation time. For values of thickness and surface acoustical reflection amplitude, paired $t$-test was used for comparison before and after enzyme digestion for each cartilage disk. $t$-test was also used to compare the extents of the change of the surface reflection amplitude induced by trypsin and collagenase. $\mathrm{P}<0.05$ was used as a significant level for the analysis of correlation or comparisons of group values. All the statistical analyses were conducted in SPSS (16.0, SPSS Inc., Chicago, IL, USA).

\section{RESULTS}

To have a sense of the reliability of the measurement, 8 repeated tests in one specimen were conducted and a mean thickness of $1.90 \pm 0.03 \mathrm{~mm}(\mathrm{CV}=1.7 \%)$ was obtained, demonstrating that the measurement of thickness was very repeatable. For the surface reflection measurement, a similar test using 8 repeated measures on one specimen harvested a value of $179.7 \pm 6.9(\mathrm{CV}=3.9 \%)$. The thickness values for all the 16 samples (Groups 1 and 2) before enzyme treatment were compared between IVUS and Vevo measurements. The thickness obtained using the two methods was highly correlated ( $r=0.985, p<0.001$, Fig. 4), indicating that the IVUS had a comparable performance with Vevo for cartilage thickness measurement. The mean thickness measured by IVUS and Vevo was $1.83 \pm 0.28 \mathrm{~mm}$ and $1.80 \pm 0.29 \mathrm{~mm}$, respectively.

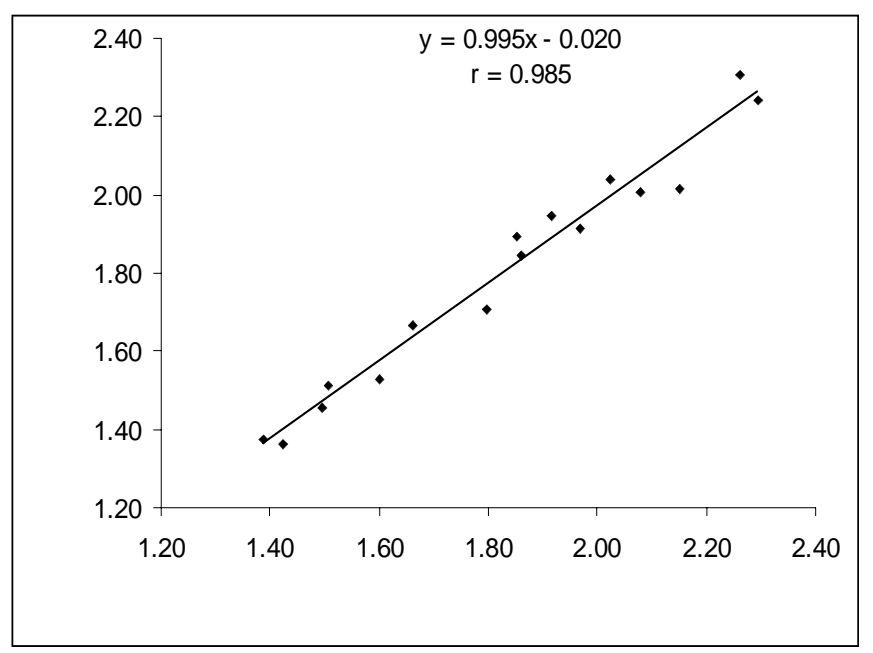

Fig. (4). A correlation of the thickness measured from IVUS and Vevo ultrasound imaging systems for cartilage specimens before enzyme digestions.

Tables $\mathbf{1}$ and $\mathbf{2}$ list the results of the thickness and surface reflection amplitude obtained by the IVUS after the treatments of trypsin and collagenase for Group 1 and Group 2 , respectively. The mean thickness value before and after treatment was $1.68 \pm 0.25 \mathrm{~mm}$ and $1.68 \pm 0.23$ for Group 1 and $1.98 \pm 0.27 \mathrm{~mm}$ and $1.99 \pm 0.26 \mathrm{~mm}$ for Group 2, respectively. Statistical analysis revealed no significant change in the sample thickness (both $p>0.05$ ) after both of the enzyme treatments. However, the surface reflection amplitude showed a significant decrease for both the treatments $(\mathrm{p}<0.01)$. The averaged decrease was $16.2 \pm$ $11.4 \%$ after trypsin digestion and $52.1 \pm 10.3 \%$ with collagenase treatment, respectively $(\mathrm{p}<0.001)$. Collagenase treatment appeared to have exerted a more significant effect on the surface reflection amplitude $(\mathrm{p}<0.001)$. The 
Table 1. The Effect of Trypsin Treatment on Articular Cartilage Measured from IVUS

\begin{tabular}{|c|c|c|c|c|}
\hline \multirow{2}{*}{ Samples } & \multicolumn{2}{|c|}{ Thickness (mm) } & \multicolumn{2}{c|}{ Surface Reflection Amplitude } \\
\cline { 2 - 5 } & Pre-treatment & Post-treatment $^{\mathbf{a}}$ & Pre-treatment $^{\text {Post-treatment }}{ }^{\mathbf{b}}$ \\
\hline \hline 1 & 1.80 & 1.79 & 175.9 & 162.2 \\
\hline 2 & 1.60 & 1.58 & 187.4 & 156.4 \\
\hline 3 & 1.39 & 1.43 & 164.4 & 137.3 \\
\hline 4 & 1.66 & 1.71 & 172.3 & 173.5 \\
\hline 5 & 2.15 & 2.06 & 188.4 & 148.6 \\
\hline 6 & 1.50 & 1.46 & 156.6 & 155.1 \\
\hline 7 & 1.85 & 1.94 & 143.2 & 143.6 \\
\hline Mean & 1.51 & 1.49 & 170.2 & 153.0 \\
\hline SD & 1.68 & 1.68 & 15.2 & 144.5 \\
\hline
\end{tabular}

${ }^{\mathrm{a}} \mathrm{p}>0.05,{ }^{\mathrm{b}} \mathrm{p}<0.01$, both paired $t$-test.

Table 2. The Effect of Collagenase Treatment on Articular Cartilage Measured from IVUS

\begin{tabular}{|c|c|c|c|c|}
\hline \multirow{2}{*}{ Samples } & \multicolumn{2}{|c|}{ Thickness (mm) } & \multicolumn{2}{|c|}{ Surface Reflection Amplitude } \\
\cline { 2 - 5 } & Pre-treatment & Post-treatment $^{\mathbf{a}}$ & Pre-treatment $^{\text {Post-treatment }}$ \\
\hline \hline 1 & 2.02 & 2.09 & 153.8 & 97.0 \\
\hline 2 & 2.26 & 2.26 & 135.9 & 73.7 \\
\hline 3 & 1.86 & 1.80 & 195.5 & 84.9 \\
\hline 4 & 1.91 & 1.91 & 175.2 & 85.0 \\
\hline 5 & 2.08 & 2.11 & 175.2 & 50.8 \\
\hline 6 & 2.29 & 2.22 & 192.3 & 78.3 \\
\hline 7 & 1.42 & 1.46 & 148.4 & 77.7 \\
\hline 8 & 1.97 & 2.08 & 160.1 & 83.1 \\
\hline Mean & 1.98 & 1.99 & 167.0 & 78.8 \\
\hline SD & 0.27 & 0.26 & 21.1 & 13.3 \\
\hline
\end{tabular}

${ }^{\mathrm{a}} \mathrm{p}>0.05,{ }^{\mathrm{c}} \mathrm{p}<0.001$, both paired $t$-test.

corresponding measurement using Vevo showed similar results: the mean pre- and post-treatment thickness for Groups 1 and 2 was $1.64 \pm 0.22$ vs. $1.65 \pm 0.22 \mathrm{~mm}(\mathrm{p}>$ $0.05)$ and $1.96 \pm 0.29$ vs. $1.95 \pm 0.25 \mathrm{~mm}(\mathrm{p}>0.05)$, respectively. The corresponding surface reflection coefficient was $3.61 \pm 0.57$ vs. $2.66 \pm 0.85(\mathrm{p}<0.01)$ and $3.64 \pm 1.11$ vs. $0.52 \pm 0.13$ ( $\mathrm{p}<0.001$ ), respectively. The surface reflection coefficient decreased by $26.9 \pm 19.5 \%$ and $84.7 \pm 4.1 \%(\mathrm{p}<0.001)$, respectively, after trypsin and collagenase treatments according to the results obtained by Vevo, showing consistent results with IVUS measurement.

\section{DISCUSSION AND CONCLUSION}

Previous researchers have used low or high frequency ultrasound to assess the articular cartilage status, which suffered from limitations such as low spatial resolution and the lack of specifically designed ultrasound transducer for arthroscopic use in vivo. In this study, we borrowed a mature technique, IVUS, from the cardiovascular field for the purpose of acquiring quantitative information of articular cartilage, taking the advantages of small size of the transducer and the use of high frequency ultrasound. The results demonstrated that the IVUS was capable of measuring the thickness and surface reflection amplitude, with the performance similar to those reported in the literature using conventional single element ultrasound transducers $[6,21,22]$. Compared to previous arthroscopic ultrasound measurement, the current IVUS will provide an extra function of tissue imaging for the cartilage. Together with its small size, it will no doubt be advantageous to incorporate this facility in the space-limited intra-articular operations.

The articular cartilage thickness change not only provides important information for the diagnosis of the osteoarthritis 
[23] but also it is a fundamental parameter for some experimental models such as the indentation test [6]. Ultrasound is regarded as a validated technique to measure the thickness of articular cartilage [24, 25]. Therefore, we evaluated the performance of IVUS by comparing with another high frequency ultrasound system in this study. A high correlation between the thickness values measured by the two systems $(r=0.985)$ demonstrated that the IVUS probe could be used to measure the cartilage thickness as conventional high frequency ultrasound. In this study, three constant speeds of sound were used for normal, trypsin and collagenase treated specimens, respectively. Although a constant sound speed such as $1610 \mathrm{~m} / \mathrm{s}$ in the current study would not deviate too much from the true value in articular cartilage, it would cause over- or underestimation of the thickness for each individual specimen. We controlled the operation of the IVUS probe in a manual way, the precise location of the probe could not be obtained and simultaneous acquisition of the speed of sound and thickness were not possible in the current study due to experiment setup. For the speed of sound issue, we adopted a different speed value for the calculations after enzyme digestions based on the values reported in previous studies [6]. Thus it was expected that the bias induced by the change of speed of sound could be partially compensated. Nevertheless, the potential error for thickness measurement caused by the uncertainty of speed of sound should be taken into account for the ultrasound measurement of articular cartilage. According to Nieminen et al (2004), the change of speed of sound in bovine articular cartilage from intact $(1603 \pm 27 \mathrm{~m} / \mathrm{s})$ to advanced (Mankin score > 4, $1548 \pm 14 \mathrm{~m} / \mathrm{s}$ ) cases in spontaneously degeneration was around $3.4 \%$ [26]. To increase the measurement accuracy, a more precise quantification of the speed of sound in the articular cartilage would be advantageous, and methods for realizing in situ measurement of the speed of sound should be further explored [18, 27, 28]. Among them, Kim et al. [28] proposed a simultaneous measurement of SOS and sample thickness using a pair of ultrasound transducers at just one side of the sample, which might be a potential method for the in-situ cartilage measurement. Another error of the thickness measurement would come from the site-dependence of the cartilage thickness [29]. To reduce the error arising from the inhomogeneous thickness of the articular cartilage, a region of about $1 \mathrm{~mm}$ in with in the center of the disk was selected for extracting the parameters. In this small region, we thought the assumption of a uniform thickness of the cartilage should be acceptable. No doubt that a better control of the measurement location would make the comparison less affected by site-dependent variation of thickness. Furthermore, comparison of thickness measured by IVUS with that measured from non-ultrasound approaches such as the histology, optical or needle probe method [25, 30], if possible, would further confirm the utility of the current measurement.

Enzyme digestion was a broadly used approach to simulate the degeneration of articular cartilage $[19,20]$. Collagenase digestion has a main effect on the breakdown of the collagen network while the trypsin has the main effect on proteoglycan (PG) digestions with minor effect on collagen network. The content of collagen and PG can be measured with histological studies. Our results showed that there was no obvious change of cartilage thickness after PG and collagen depletions. Our finding was in agreement with those reported previously. In a study using needle probe measurement of the cartilage thickness, PG and collagen depletions also caused slight but not significant decrease in the thickness [21]. Wang et al. [30] reported that the thickness of cartilage measured using ultrasound, digital caliper and histology methods did not change significantly after trypsin digestions. Although no significant change in the cartilage thickness resulted from trypsin and collagenase treatment, the cartilage would behave differently from its normal status because its structure had been varied significantly as observed from the surface ultrasound reflection measurement [31] or other mechanical tests [6, 10]. For the measurement of the surface reflection amplitude, because the penetration angle of the acoustic beam would exert significant impact on the reflection level, it was important to adjust the orientation of the probe to acquire signal amplitude as large as possible to ensure the perpendicular incidence of ultrasound beam. This had been paid attention to throughout the experiments using IVUS and Vevo systems. It was revealed from the current measurement that both the treatments resulted in significant decreases in the surface reflection amplitude with a more significant impact from the collagenase, which was also consistent with the results of previous studies $[6,12,21]$. As the surface reflection amplitude was mainly determined by the surface collagen network integrity, breakdown of the collagen network would cause a far more significant effect on the parameter compared with PG digestion. Thus this study further added to the literature that ultrasound could be used as a potential method to differentiate the cartilage degeneration induced by collagen or PG depletions [6, 21]. Histological/histochemical analysis of the collagen and $\mathrm{PG}$ contents with and without enzyme digestions would further confirm the utility of the current IVUS measurement.

It should be noted in the current study that the extraction of the thickness and surface reflection coefficient by the IVUS measurement on cartilage was performed directly from the B-mode image, which had lost parts of its information and the spatial resolution compared to the originally obtained ultrasound signals in the RF format as the signals in the Vevo measurement. The results from this study showed that the decrease of surface reflection amplitude measured using IVUS after collagenase digestion (52.1 \pm $10.3 \%$ ) was much smaller than that obtained by Vevo $(84.7$ $\pm 4.1 \%)$. The main reason for this may be a nonlinear mapping from the RF data to B-mode image data in the IVUS system. Directly including the RF signals and extracting more intrinsic material parameters were recommended in future applications for enhancing the capabilities and sensitivity of the measurement using IVUS [32].

In conclusion, the feasibility of using IVUS as a potential tool for the quantitative assessment of articular cartilage was demonstrated in this study. For further development of this method, IVUS-integrated probe in the arthroscopy-based instrument is currently being designed to facilitate an endoscopic evaluation of the cartilage status, for example, the water-jet indentation probe may be integrated with IVUS for measuring the morphological, acoustical and mechanical 
properties and elastographic imaging of the articular cartilage in osteoarthritis [33-39].

\section{ACKNOWLEDGEMENTS}

The authors would like to thank Dr. William Hau and Mr. Eddie Mak for supporting us the IVUS system for conducting the measurement. Thanks also go to Miss Wang Shu-zhe and Mr Cheung Chung-wai James for preparing the articular cartilage samples used in this study. This study is partially supported by The Hong Kong Research Grant Council (PolyU5318/05E, PolyU5354/08E) and The Hong Kong Polytechnic University (J-BB69).

\section{REFERENCES}

[1] A. M. Aisen, W. J. McCune, A. MacGuire, P. L. Carson, T. M. Silver, S. Z. Jafri, and W. Martel, "Sonographic evaluation of the cartilage of the knee," Radiology, vol. 153, pp. 781-784, 1984.

[2] I. Moller, D. Bong, E. Naredo, E. Filippucci, I. Carrasco, C. Moragues, and A. Iagnocco, "Ultrasound in the study and monitoring of osteoarthritis," Osteoarthr. Cartil., vol. 16, pp. S4S7, 2008.

[3] W. Grassi, G. Lamanna, A. Farina, and C. Cervini, "Sonographic imaging of normal and osteoarthritic cartilage," Semin. Arthritis Rheum., vol. 28, pp. 398-403, Jun 1999.

[4] L. Friedman, K. Finlay, and E. Jurriaans, "Ultrasound of the knee," Skeletal Radiol., vol. 30, pp. 361-377, Jul 2001.

[5] D. E. T. Shepherd, and B. B. Seedhom, "Thickness of human articular cartilage in joints of the lower limb," Ann. Rheum. Dis., vol. 58, pp. 27-34, Jan 1999.

[6] M. S. Laasanen, J. Toyras, J. Hirvonen, S. Saarakkala, R. K. Korhonen, M. T. Nieminen, I. Kiviranta, and J. S. Jurvelin, "Novel mechano-acoustic technique and instrument for diagnosis of cartilage degeneration," Physiol. Meas., vol. 23, pp. 491-503, Aug 2002.

[7] K. Hattori, Y. Takakura, M. Ishimura, T. Habata, K. Uematsu, and K. Ikeuch, "Quantitative arthroscopic ultrasound evaluation of living human cartilage," Clin. Biomech., vol. 19, pp. 213-216, 2004.

[8] I. M. Rutkow, "Surgical operations in the United States - then (1983) and now (1994)," Arch. Surg., vol. 132, pp. 983-990, Sep 1997.

[9] D. Kane, D. J. Veale, O. FitzGerald, and R. Reece, "Survey of arthroscopy performed by rheumatologists," Rheumatology, vol. 41, pp. 210-215, Feb 2002.

[10] Y. P. Zheng, C. X. Ding, J. Bai, A. F. T. Mak, and L. Qin, "Measurement of the layered compressive properties of trypsintreated articular cartilage: an ultrasound investigation," Med. Biol. Eng. Comput., vol. 39, pp. 534-541, Sep 2001.

[11] P. Kiviranta, E. Lammentausta, J. Toyras, I. Kiviranta, and J. S. Jurvelin, "Indentation diagnostics of cartilage degeneration," Osteoarthr. Cartil., vol. 16, pp. 796-804, 2008.

[12] E. Cherin, A. Saied, P. Laugier, P. Netter, and G. Berger, "Evaluation of acoustical parameter sensitivity to age-related and osteoarthritic changes in articular cartilage using $50-\mathrm{MHz}$ ultrasound," Ultrasound Med. Biol., vol. 24, pp. 341-354, March 1998.

[13] R. S. Adler, D. K. Dedrick, T. J. Laing, E. H. Chiang, C. R. Meyer, P. H. Bland, and J. M. Rubin, "Quantitative assessment of cartilage surface-roughness in osteoarthritis using high-frequency ultrasound," Ultrasound Med. Biol., vol. 18, pp. 51-58, 1992.

[14] H. K. W. Kim, P. S. Babyn, K. A. Harasiewicz, H. K. Gahunia, F. P. H. Pritzker, and F. S. Foster, "Imaging of immature articular cartilage using ultrasound backscatter microscopy at $50 \mathrm{MHz}, " J$. Orthop. Res., vol. 13, pp. 963-970, Nov 1995.

[15] M. P. Spriet, C. A. Girard, S. F. Foster, K. Harasiewicz, D. W. Holdsworth, and S. Laverty, "Validation of a $40 \mathrm{MHz}$ B-scan ultrasound biomicroscope for the evaluation of osteoarthritis lesions in an animal model," Osteoarthr. Cartil., vol. 13, pp. 171179, Feb 2005.

[16] K. M. Ziada, S. R. Kapadia, E. M. Tuzcu, and S. E. Nissen, "The current status of intravascular ultrasound imaging," Curr. Probl. Cardiol., vol. 24, pp. 547-616, September 1999.
[17] O. Gulel, I. Sipahi, and E. M. Tuzcu, "Intravascular ultrasound: questions and answers," Anadolu. Kardiyol. Derg., vol. 7, pp. 169178, June 2007.

[18] S. G. Patil, Y. P. Zheng, J. Y. Wu, and J. Shi, "Measurement of depth-dependence and anisotropy of ultrasound speed of bovine articular cartilage in vitro," Ultrasound Med. Biol., vol. 30, pp. 953-963, July 2004.

[19] E. D. Harris, E. L. Radin, S. M. Krane, and H. G. Parker, "Effects of proteolytic-enzymes on structural and mechanical properties of cartilage," Arthritis Rheum., vol. 15, pp. 497-503, 1972.

[20] W. D. Shingleton, D. J. Hodges, P. Brick, and T. E. Cawston, "Collagenase: a key enzyme in collagen turnover," Biochem. Cell Biol., vol. 74, pp. 759-775, Sep 1996.

[21] J. Toyras, J. Rieppo, M. T. Nieminen, H. J. Helminen, and J. S. Jurvelin, "Characterization of enzymatically induced degradation of articular cartilage using high frequency ultrasound," Phys. Med. Biol., vol. 44, pp. 2723-2733, Nov 1999.

[22] K. Hattori, K. Mori, T. Habata, Y. Takakura, and K. Ikeuchi, "Measurement of the mechanical condition of articular cartilage with an ultrasonic probe: quantitative evaluation using wavelet transformation," Clin. Biomech., vol. 18, pp. 553-557, July 2003.

[23] R. L. Karvonen, W. G. Negendank, R. A. Teitge, A. H. Reed, P. R. Miller, and F. Fernandezmadrid, "Factors affecting articularcartilage thickness in osteoarthritis and aging," J. Rheumatol., vol. 21, pp. 1310-1318, July 1994.

[24] V. E. Modest, M. C. Murphy, and R. W. Mann, "Optical verification of a technique for in-situ ultrasonic measurement of articular-cartilage thickness," J. Biomech., vol. 22, pp. 171-176, 1989.

[25] J. S. Jurvelin, T. Rasanen, P. Kolmonen, and T. Lyyra, "Comparison of optical, needle probe and ultrasonic techniques for the measurement of articular-cartilage thickness," J. Biomech., vol. 28, pp. 231-235, Feb 1995.

[26] H. J. Nieminen, S. Saarakkala, M. S. Laasanen, J. Hirvonen, J. S. Jurvelin, J. Toyras, "Ultrasound attenuation in normal and spontaneously degenerated articular cartilage", Ultrasound. Med. Biol., vol. 30, pp. 493-500, 2004.

[27] J. K. F. Suh, I. Youn, and F. H. Fu, "An in situ calibration of an ultrasound transducer: a potential application for an ultrasonic indentation test of articular cartilage," J. Biomech., vol. 34, pp. 1347-1353, Oct 2001.

[28] Y. H. Kim, S. J. Song, and J. K. Lee, "Simultaneous measurements of the ultrasonic wave velocity and thickness of a solid plate made from one side of the plate," Meas. Sci. Technol., vol. 14, pp. N13N16, May 2003.

[29] C. Adam, F. Eckstein, S. Milz, E. Schulte, C. Becker, and R. Putz, "The distribution of cartilage thickness in the knee-joints of oldaged individuals - measurement by A-mode ultrasound," Clin Biomech., vol. 13, pp. 1-10, Jan 1998.

[30] Q. Wang, Y. P. Zheng, L. Qin, Q. H. Huang, W. L. Lam, G. Leung, $\mathrm{X}$. Guo, and H. B. Lu, "Real-time ultrasonic assessment of progressive proteoglycan depletion in articular cartilage," Ultrasound Med. Biol., vol. 34, pp. 1085-1092, 2008.

[31] S. Saarakkala, J. Toyras, J. Hirvonen, M. S. Laasanen, R. Lappalainen, and J. S. Jurvelin, "Ultrasonic quantitation of superficial degradation of articular cartilage," Ultrasound Med. Biol., vol. 30, pp. 783-792, Jun 2004.

[32] Y. P. Zheng, and Y. P. Huang, "More intrinsic parameters should be used in assessing degeneration of articular cartilage with quantitative ultrasound," Arthritis. Res. Ther., vol. 10, p. 125, 2008.

[33] M. H. Lu, Y. P. Zheng, and Q. H. Huang, "A novel noncontact ultrasound indentation system for measurement of tissue material properties using water jet compression," Ultrasound Med. Biol., vol. 31, pp. 817-826, June 2005.

[34] M. H. Lu, Y. P. Zheng, and Q. H. Huang, "A novel method to obtain modulus image of soft tissues using ultrasound water jet indentation: a phantom study," IEEE Trans. Biomed. Eng., vol. 54, pp. 114-121, Jan 2007.

[35] Y. P. Zheng, S. L. Bridal, J. Shi, A. Saied, M. H. Lu, B. Jaffre, A. F. T. Mak, and P. Laugier, "High resolution ultrasound elastomicroscopy imaging of soft tissues: system development and feasibility," Phys. Med. Biol., vol. 49, pp. 3925-3938, Sep 2004

[36] D. T. Ginat, G. Hung, T. R. Gardner, and E. E. Konofagou, "Highresolution ultrasound elastography of articular cartilage in vitro," in Proceedings of the 28th IEEE EMBS Annual International Conference, Aug 30-Sep 3, New York, 2006, pp. 6644-6647. 
[37] P. A. Hardy, A. C. Ridler, C. B. Chiarot, D. B. Plewes, and R. M. Henkelman, "Imaging articular cartilage under compressioncartilage elastography," Magn. Reson. Med., vol. 53, pp. 10651073, May 2005.

[38] C. L. de Kortem, and A. F. W. van der Steen, "Intravascular ultraso-und elastography: an overview," Ultrasonics, vol. 40, pp. 859-865, July 2002.
[39] M. H. Lu, Y. P. Zheng, Q. H. Huang, C. Ling, Q. Wang, L. Bridal, L. Qin, and A. Mak, "Noncontact evaluation of articular cartilage degeneration using a novel ultrasound water jet indentation system," Ann. Biomed. Eng., vol. 37, pp. 164-175, Jan 2009.

Received: January 09, 2009

(C) Huang and Zheng; Licensee Bentham Open.

This is an open access article licensed under the terms of the Creative Commons Attribution Non-Commercial License (http://creativecommons.org/licenses/by-nc/3.0/) which permits unrestricted, non-commercial use, distribution and reproduction in any medium, provided the work is properly cited. 